\title{
Tout ce qui fait de l'effet a aussi des effets secondaires
}

\section{Christoph Bosshard}

Dr med., vice-président et membre du Comité central de la FMH, responsable du département Données, démographie et qualité / Académie suisse pour la qualité en médecine (ASQM)

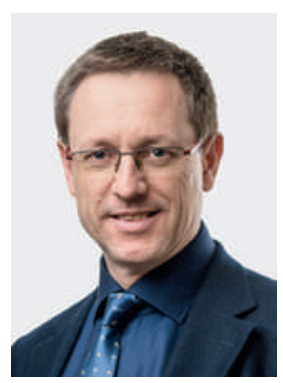

Depuis Paracelse, nous savons que les effets souhaités arrivent rarement sans effets secondaires et nous appliquons inlassablement cette théorie dans notre quotidien de médecin. Nous examinons toujours le bénéfice, les risques et les effets secondaires pour nos patients, et recherchons avec eux la solution qui correspond le mieux à leur besoin. Le Bulletin des médecins suisses a déjà plusieurs fois relaté les réflexions intensives menées par le corps médical à ce sujet et je me permets de vous rappeler les publications sur la décision partagée (Shared Decision Making), les guidelines et les indicateurs de qualité.

\section{Peser le bénéfice et les risques d'un traitement et trouver la meilleure solution pour et avec le patient sont au cœur de notre activité de médecin.}

Les patients qui nous demandent le plus d'attention font généralement face à des défis complexes conjuguant problèmes de santé et contexte psychosocial. Leur situation est toujours unique. Un remède miracle serait un soulagement pour tout le monde. Seulement voilà, il n'existe pas de tel remède, tout aussi peu qu'il n'existe de patient standard.

Combien de médecine les patients veulent-ils? Et notre société, quel système de santé peut-elle se permettre? A la première question, nous ne pouvons généralement répondre qu'après le traitement concret du patient, et ce uniquement dans le contexte des décisions prises et du chemin parcouru. $\mathrm{Au}$ cas par cas, il est hypothétique de vouloir savoir si une autre décision, une autre voie eût été meilleure. Aujourd'hui, de plus en plus de questions sont traitées du point de vue médical alors que notre société devrait avoir le courage de les discuter et de les trancher dans un contexte général. Peut-être aussi que la tendance à la médicalisation repose sur la confiance illimitée que les citoyens vouent à leur système de santé, un système qu'ils plébiscitent et qui occupe régulièrement les meilleures places en comparaison internationale. Le besoin de sécurité est également un facteur qu'il ne faut pas sous-estimer. Et pourtant, notre système peut-il se permettre tout ça? A quels effets secondaires faut-il s'attendre? Personne ne sera surpris d'apprendre qu'en présence d'une bonne prise en charge médicale et d'une bonne accessibilité aux soins, le système tendra à la surconsommation de prestations médicales, et inversement, une couverture lacunaire le fera tendre vers la sous-consommation.

Le document-cadre que nous présentons dans ce numéro du Bulletin des médecins suisses (page 236) se penche en premier lieu sur le thème de la surconsommation, bien que les autres aspects ne soient pas moins importants. D'autres questions se posent aussi sous la forme de notions telles que la prise en charge minimale, maximale ou optimale. Mais où commence la surconsommation, quand les effets secondaires l'emportent-ils sur le bénéfice? Comment se différencie la réflexion au niveau du système en général et de l'individu en particulier? Jusqu'où peut aller la solidarité de la communauté dans le cadre des assurances sociales? Où ont lieu les transferts de coûts et comment le savoir sans un aperçu économique global à long terme puisque le bénéfice n'apparaît pas là où les coûts sont générés? Pourquoi parlons-nous toujours de coûts et si rarement de bénéfice?

La surconsommation et la sous-consommation de prestations médicales sont finalement aussi des questions de portée sociétale.

Toutes ces questions, nous, médecins, devons aussi nous les poser afin de faire partager activement nos expériences et notre point de vue. En définitive, le thème de la surconsommation ou de la sous-consommation a une portée également sociétale. Notre document-cadre vise à susciter et à soutenir la discussion sur la base de l'évidence scientifique actuelle mais aussi à en montrer les limites. Là aussi, je vous invite à soutenir la FMH par votre collaboration active au sein de vos organisations de base et de vos sociétés de discipline. 